\title{
Let's Know about Community Based Health Care (CBHC) in Afghanistan
}

\author{
Said Habib Arwal*
}

National Coordinator of $C B H C$, Afghanistan

*Corresponding author: Said Habib Arwal, National Coordinator of CBHC, Afghanistan, E-mail: saidhabiba@gmail.com

Receiving date: August 07 2015; Accepted date: August 31 2015; Published date: September 12015

Copyright: (C) 2015 Arwal SH. This is an open-access article distributed under the terms of the Creative Commons Attribution License, which permits unrestricted use, distribution, and reproduction in any medium, provided the original author and source are credited.

\section{Short Communication}

Afghan's CBHC program is a best modal of three way (community, NGOs and Government) Partnership, for community engagement and empowerment to improve their Health by their active participation.

Community-based health care ( $\mathrm{CBHC})$ is the cornerstone of successful implementation of the Health system in Afghanistan. It provides the context for the most comprehensive interaction between the health system and the communities it serves. Its success depends upon community participation and a partnership between community and health staff.

$\mathrm{CBHC}$ is not new to Afghanistan; it existed prior to the many years of war and conflict. However, in this post conflict period, Afghanistan has reviewed the international concepts and developed an Afghanistan-specific form of $\mathrm{CBHC}$, which was adopted by the Ministry of Health following a national conference on CBHC in September 2002.

The implementation of $\mathrm{CBHC}$ recognizes first that families and communities have always looked after their own health. Religion and cultural norms and beliefs play an important part in health practices, and families are making decisions to maintain health or care for illness every day. In addition, community members understand and have better information on local needs, priorities, and dynamics. The partnership of health services with communities, therefore, has two aspects:

- To persuade families and communities to make appropriate use of scientific health services, and to change certain behaviours and social norms for more healthy ones,

- To accept the guidance and collaboration of communities in the implementation of health programs and the acceptable provision of health care, and encourage them to identify and solve their own problems.

While there is no universally accepted definition of CBHC, global experience has identified three consistent components of CBHC:

- Partnership between the community and the health facility staff,

-Appropriate and good quality care by community-based providers,

- Promotion of healthy practices and life styles.

Experience of the implementation of these components has produced a set of global principles of CBHC:

- $\mathrm{CBHC}$ focuses on major health problems for which solutions exist.

- The lowest-level health worker can provide the service at a reasonable standard of quality.

- Health workers are locally identified and recruited.

- Health workers are trained incrementally, one skill at a time.
- An established list of drugs and supplies is used.

- Supervision is regular and supportive.

- The health worker is accountable to the community.

- The community makes a financial or in-kind contribution for the services.

The components of the Afghan CBHC system include community health workers, community health supervisors, health shuras, family health action groups and family health workers:

CHWs are community volunteers. A health post has one male and one female $\mathrm{CHW}$ to serve a population of 100-150 families. The $\mathrm{MoPH}$ encourages the training of couples assigned to the same health post. At least $40 \%$ of CHWs should be female. A health post serves a population of between 1000 and 1500 people, but in sparsely populated areas the population served may be as small as 400 . Health program managers should give special attention to the coverage of communities by CHWs and train more if required to give access to the whole population. CHWs are trained to provide high impact primary care in the community, and to refer severely sick patients and those needing preventive services or delivery care. They also promote healthy behaviours and lifestyles in the community. CHWs maintain records of their activities and provide monthly reports to the HMIS system.

The community shura provides leadership and support to all health-related activities in their community. They select, support and supervise the CHWs in the community; they monitor the community map with the CHWs to be able to encourage families to make full use of preventive health services including outreach services; they provide leadership in the adoption and promotion of new behaviours and social norms.

Community Health Supervisors are members of the health facility staff. They are the main links between the facility and the communities around the facility. They support and supervise all the CHWs, collect and process all monthly reports from $\mathrm{CHWs}$, meet regularly with Shura, and manage all community-based health programs.

It is a support group to female CHWs whose aim is to improve the life style and appropriate use of health services for reducing the mortality rate of mothers and children. With the formation of these groups from the community members, they are services are utilized for spreading health awareness and key health messages among mothers. This will result in healthy homes and healthy lifestyles and will increase the use of available health services. It will build a strong information and referral system within the community that will help CHWs attend to pregnancies, births, and other cases of illness.

Female CHWs select a group of 10-15 women with young families, respected within their community, and share with them what they have learned. These 10-15 women groups in turn promote adoption of 
healthy behaviours among the women in their neighbouring households. It is expected that this will make the work of the CHWs more effective and more efficient, even reducing their current workload.

To further strengthen the CBHC Program and improve health status of the populations, Ministries of Public Health and Education are determined to impart health education to school students and enable them to get health messages to their families and play the role of health educators. This program will start from grade one and continue till graduation from high school. As a result, these graduates will be awarded CWHs' certificates with a difference that they have not received treatment related training and are not authorized to prescribe medicines.

It is expected that this project will improve the health status of the people of Afghanistan, especially mothers and children under five, particularly in remote areas and improve the health system quality and strengthen the stewardship role of the Ministry of Public Health and consequently lead to the improvement of general health, social and economic status of the people.

CBHC department also have significant achievements some are as below:

Strengthened the CBHC System (near 29000 volunteer CHWs, more than 16000 shura-e-sehi, more than 5000 FHAG, 1245 CHS, strong relation with CM \& CHNE),

Scaled up a community-based Family Planning Program,

Establish a strong team at the CBHC department,

Built the CBHC staff capacity through trainings, mentoring, and study tours,

Improving coordination and advocacy with partners.

\section{Improve CBHC Monitoring Mechanism}

Developed monitoring tools,

Conducted frequent monitoring and post-training visits,

Set a follow up system,

Conducted 30 monitoring visits from community level since Sept. 2012.

\section{Developing policies, strategies, guidelines and protocols}

National strategy for CBHC in 2009 and revised it in 2014,

CBHC strategies for poor urban and nomads,

Training manuals for CHSs' initial and in-service trainings,

National CBHC Package,

Training manual for DHOs,

Recognition mechanisms for CHWs,

New policy that allows CHWs to give first dose of DMPA,

Booklet on FP in the light of Islam,
Training curriculum for school children to work as Family Health Workers,

Revision of CHWs' Manual,

Revision of CHWs' Kit,

Developed Guideline and manual for Health Shura members to enhance their governance and leadership role,

Piloted two new best practices (IHP and Cleaning Friday) in four provinces,

Strengthening referral system from community to health facilities,

Initiate CBHC programs for the urban poor and nomads,

Expanded FHA Groups in the country,

Simplified community mapping,

Piloted two new best practices (IHP and Cleaning Friday) in four provinces,

Strengthening referral system from community to health facilities,

Initiate CBHC programs for the urban poor and nomads,

Expanded FHA Groups in the country,

Simplified community mapping.

\section{CBHC also trained}

177 provincial CBHC officers, NGOs CBHC officers and CHWs trainers by conducting seven TOTs on CHWs revised manual,

$103 \mathrm{CBHC}$ officers and CHW trainers by conducting three TOTs on CHSs' initial training manual,

107 CBHC officers and CHWs trainers by conducting 5 TOTs training on First Aid,

151 CBHC officers and CHWs trainers by conducting three TOTs on FHA group manual,

22 CBHC officers on by conducting a TOT on LMG and M\&E,

258 Health Shura members on newly developed Guideline,

1017 CHSs on CHSs' initial training manual through NGOs,

22 female CHSs and 278 female CHWs by conducting refresher trainings on CBHC, HIV. Malaria and TB,

27 DHOs by conducting a TOT on CBHC.

\section{CBHC also conducted}

The National CHWs Conference,

The National Community Shuras' Workshop,

The CHSs' National Coordination Workshop,

The CBHC Consensus Building Workshop,

Seven regional advocacy workshops,

Family Health Action Group National workshops. 